\title{
Penicillium Marneffei Infection Presenting as Vulv-ulcer IN AN HIV-INFECTED WOMAN
}

\author{
Y. Cui ${ }^{1,2}$, C. Jin ${ }^{1}, \mathrm{X} . \mathrm{Li}^{3}, \mathrm{~N} . \mathrm{Wu}^{1}$ \\ ${ }^{1}$ State Key Laboratory for Diagnosis and Treatment of Infectious Disease, First Affiliated Hospital, School of Medicine, \\ Zhejiang University, Hangzhou, Zhejiang, China \\ ${ }^{2}$ Department of Dermatovenereology, The Sixth Hospital Affiliated to Zhejiang University of Traditional Chinese Medicine, \\ Hangzhou, Zhejiang, China \\ ${ }^{3}$ Microbiology Lab, The Sixth Hospital Affiliated to Zhejiang University of Traditional Chinese Medicine, Hangzhou, Zhejiang, China
}

\section{Abstract}

We report the case of an HIV-infected patient with vulv-ulcer as first manifestation associated with Penicillium marneffei, which is of special interest for healthcare workers in all fields.

\section{CASE REPORT}

A 23-year-old woman was admitted to our department with the chief complaint of an 1-month-history of painful vulv-ulcer and a two-week fever. She reported an 1-month-history of vulv-ulcer and expanded in the past two weeks. Initially rapid plasma reagin circle card test (RPR) and treponema palidum hemaglutination assay (TPHA) were detected positively, and treated with benzylpenicillin with no improvement. Physical examinations showed lymphadenopathy, swelling and tenderness, in supraclavicular, axillary and inguinal. On both sides of the labia majora there were ulcers, well-defined and slightly swelled up, palpated softly and painly. His hemogram values at admission were a leukocyte count $=6.3 \times 10^{3} \mathrm{cells} / \mu \mathrm{l}($ normal range $=$ $4-10 \times 10^{3}$ cells $\left./ \mu \mathrm{l}\right)$ comprising $92.4 \%$ neutrophils (normal range $=50 \%-70 \%$ ), hemoglobin level $=$ $7.6 \mathrm{~g} / \mathrm{dl}$ (normal range $=11-15 \mathrm{~g} / \mathrm{dl}$ ); Blood chemistry data showed an aspartate aminotransferase level of $176 \mathrm{U} / \mathrm{L}$ (normal range $=0-50 \mathrm{U} / \mathrm{L}$ ), an alanine aminotransferase level of 91U/L (normal range = $0-50 \mathrm{U} / \mathrm{L}$ ). RPR was negative and TPHA was positive. Herpes simplex virus II and hepatitis virus were negative. The patient was serologically positive for HIV and her $\mathrm{CD}_{4}{ }^{+} \mathrm{T}$ lymphocyte count was 8 cells $/ \mu \mathrm{l}$. Autologous antibody was negative. Ulcer secretions and blood culture demonstrated Penicillium marneffei positive and Gram negative bacilli negative. A chest radiograph showed normal. Biopsy was unavailable for patient's refusal. The patient received intravenous voriconazole for 6 days of treatment $(6 \mathrm{mg}$ per kilogram intravenously twice a day on day 1 , followed by 4 mg per kilogram intravenously twice daily for 5 days), the temperature decreased to normal and tenderness of Ulcer lightened and discharged with Itraconazole for the patient's requirement. The patient was still alive and not recurrent.

\section{Discussion}

Penicilliosis marneffei, an opportunistic infectious disease caused the dimorphic fungus Penicilliosis marneffei that grows as a mold at $25^{\circ} \mathrm{C}$ and as a yeast at $37^{\circ} \mathrm{C}$ and has emerged as the third most common opportunistic pathogen among HIV-positive individuals in Southeast Asia [1]. The clinical manifestations of disseminated $P$. marneffei infection are non-specific and include fever, anaemia, generalized lymphadenopathy and hepatomegaly. Cutaneous manifestations may provide a clue to the diagnosis-typically, umbilicated papules with or without central necrosis (molluscumcontagiosum-like) distributed on the upper trunk, upper extremities, face and scalp [2].

Although no anti-fungal agents have received regulatory approval specifically for systemic Penicillium marneffe $i$ infection, response to anti-fungal therapy is good if the treatment is started early, and amphotericin B and itraconazole are generally effective [3].

Voriconazole is a new extended-spectrum triazole anti-fungal agent with potent in vitro activity against a range of molds, yeasts, and dematiaceous fungi [4]. The in vitro activities of voriconazole against Penicillium marneffei are superior to those of amphotericin B and comparable with those of itraconazole; voriconazole minimum inhibitory concentrations (determined using NCCLS and Etest methods) were in the range of $<0.01-0.06 \mu \mathrm{g} / \mathrm{mL}$, and minimum fungicidal concentrations were $0.12-0.5 \mu \mathrm{g} / \mathrm{mL}$ [5].

The results from these studies suggest that voriconazole is an effective therapeutic option for AIDS patients with systemic Penicillium marneffei infection.

The case we described is of note for several reasons. (1) Although Genital ulcer caused by Penicillium marneffei has been described once previously [6], to our knowledge, genital ulcer caused by Penicillium marneffe $i$ in an HIV patient is extremly rare. (2) In our patient the vulv-ulcer was initially labeled as 'syphilis' because the patient had positive treponemal serologic tests (3). The ulcer seemed like chancroid, but secretion culture was Penicillium marneffei positive;(4) The mortality rate in patients with acute disseminated 
penicilliosis marneffei is high if the infection is not diagnosed early enough and a timely and effective antifungal therapy is not used.

\section{REFERENCES}

1. Supparatpinyo K., Khamwan C, Baosoung V, Nelson KE, Sirisanthana T. Disseminated Penicillium marneffei infection in Southeast Asia. Lancet. 1994 Jul 9;344(8915): 110-3.

2. Vanittanakom N, Sirisanthana T. Penicillium marneffei infection in patients infected with human immunodeficiency virus. Curr Top Med Mycol. 1997 Dec;8(1-2):3542. Review.

3. Duong TA. Infection due to Penicillium marneffei, an emerging pathogen: Review of 155 reported cases. Clin Infect Dis. 1996 Jul;23(1):125-30. Review.

4. Espinel-Ingroff A. In vitro fungicidal activities of voriconazole, itraconazole, and amphotericin B against opportunistic moniliaceous and dematiaceous fungi. J Clin Microbiol. 2001 Mar;39(3):954-8.

5. Espinel-Ingroff A, Steele-Moore L. In vitro activities of voriconazole against the mycelial and yeast forms of Penicillium marneffei by different testing conditions. Mycoses. 2001; 44: 17.
6. Annam V, Inamadar AC, Palit A, Koppad M, Peerapur BV, Yelikar BR. Genital ulcer caused by Penicillium marneffei in an HIV-infected patient. Sex Transm Infect. 2007 Jun; 83(3):249-50.

Received: May 2, 2011 / Accepted: May 16, 2011

Address for correspondence:

Nanping Wu

State Key Laboratory for Diagnosis and Treatment of Infectious Disease

First Affiliated Hospital

School of Medicine

Zhejiang University

Hangzhou, Zhejiang 310003

China

Tel. 0086-571-87236580

E-mail: flwnp@yahoo.com.cn 